\title{
Proper studies of selective serotonin reuptake inhibitors are needed for youth with depression
}

\section{Robert Gibbons PhD, J. John Mann MD}

$\infty \infty$

See related research paper by Barbui and colleagues, page 291
$\mathrm{E}$ vidence of a possible association between the use of selective serotonin reuptake inhibitors (SSRIs) and suicide in youth presents a vexing problem for clinicians, youth and their families. Are these medications the most effective treatment for major depression in children, adolescents and young adults? Do SSRIs carry a greater risk for suicide than no treatment or alternative treatments such as other antidepressants or specific psychotherapies?

In this issue, Barbui and colleagues ${ }^{1}$ report the results of a meta-analysis of 8 large-scale observational studies that compared the risk of suicide among patients with depression who received SSRIs and those with no exposure to antidepressants. In contrast to the results reported from a meta-analysis performed by the US Food and Drug Administration (FDA), which concluded that there was a neutral effect of SSRIs on the risk of suicide among adults aged 25-64 years, Barbui and colleagues report a protective effect of SSRIs in this age group. Furthermore, although the FDA concluded that there was increased risk of suicidality associated with the use of antidepressants among youth aged 18-24 years and extended the "black-box" warning for use of antidepressants to this age group, Barbui and colleagues found a beneficial effect. Of interest, a study by Gibbons and colleagues, ${ }^{2}$ which was included in the analysis by Barbui and colleagues, included a large sample of patients with major depression from Veterans' Affairs centres. This study specifically examined the effect of SSRIs in patients aged 18-25 years. Gibbons and colleagues found a protective effect of antidepressants in terms of lower rates of suicide attempts compared to patients who received no treatment. They also reported a protective effect of SSRIs when the risk of suicide was compared in the same cohort before and during treatment.

Barbui and colleagues found a higher rate of suicide attempts and completions among adolescents and a lower rate among adults whose depression was treated with SSRIs. These results are reassuring for adults and their clinicians and families, but worrisome for adolescents and their clinicians and families. However, we must reconcile these findings with other conflicting data. Sondergard and colleagues ${ }^{3}$ noted that none of the 42 youth in Denmark aged 10-17 years who committed suicide between 1995 and 1999 had taken an SSRI within 2 weeks before their suicide. If SSRIs increase the risk of suicide, it is unclear why these drugs are so rarely found on toxicological examination of youth after suicide. In addition, in youth, suicide appears to occur most commonly among those with untreated mood disorders. The epidemiologic studies of Gibbons and colleagues ${ }^{4}$ and Olfson and colleagues, ${ }^{5}$ both of which used national US rates of suicide

\section{Key points}

- The conclusion by Barbui and colleagues that SSRIs are protective against suicidality among young adults challenges the justification of a "black box" warning about the risk of suicide with the use of SSRIs.

- Their meta-analysis confirms the results of previous FDA analyses that there is an increased risk of ideation and nonfatal suicidal behaviour among children and adolescents who use SSRIs.

- Randomized controlled trials that include adolescents with depression and that use more sensitive measures than previous studies are needed to prospectively test the effectiveness and safety of SSRIs.

among youth, reported a correlation between lower prescription rates of SSRIs and higher suicide rates among both children and adults. Moreover, since the decrease in prescription rates in 2004 and 2005 among adolescents, ${ }^{6}$ there has been an increase in the suicide rate in that age group. ${ }^{7}$ Finally, observed differences between adolescents and adults in the association of the use of SSRIs and suicide may reflect confounding by severity of illness that may result from greater difficulty in matching treated and untreated adolescents because their historical record of illness is shorter than for adults. In this respect, studies that compare the rate of suicide attempts before and after treatment initiation within the same cohort may have an advantage over studies comparing rates in different cohorts.

Alarmingly, concerns about the risk of suicide in youth have led not only to fewer SSRI prescriptions without substitution of alternative medications or psychotherapies, but also to a decrease in predicted rates of diagnosis of mood disorders. ${ }^{8}$ Since a decrease in the rate of true depression is improbable, clinicians may be avoiding making this diagnosis to avoid the dilemma of whether to prescribe antidepressants to children and adolescents. With so many uncertainties, large randomized trials are now required. These are not impossible to carry out. Randomized controlled studies of the use of lithium for treatment of mood disorders and the use of clozapine for treatment of schizophrenia have shown that these drugs have antisuicidal therapeutic effects relative to comparators. Similar studies of SSRIs that include suicidal adolescents with major depression are urgently needed to determine the safety and efficacy of these medications.

Robert Gibbons is with the Center for Health Statistics, University of Illinois at Chicago, Chicago, USA. J. John Mann is with the Division of Molecular Imaging and Neuropathology, Department of Psychiatry, Columbia University, and the New York State Psychiatric Institute, New York, USA. 
Competing interests: Robert Gibbons has been an expert witness in suiciderelated cases for Wyeth, Pfizer and the US Department of Justice. J. John Mann holds a research grant from GlaxoSmithKline for a positron emission tomography study of amyloid protein levels in the brains of patients with Alzheimer disease and mild cognitive impairment. He also holds a research grant from Novartis for a positron emission tomography study of mGluR5 receptors in depression. He receives no salary support or personal payments from these grants.

Contributors: Both of the authors contributed to the content of this article, revised it critically and approved the final version submitted for publication.

\section{REFERENCES}

1. Barbui C, Esposito E, Cipriani A. Selective serotonin reuptake inhibitors and risk of suicide: a systematic review of observational studies. CMAJ 2009; 180:291-7.

2. Gibbons RD, Brown $\mathrm{CH}$, Hur K, et al. Relationship between antidepressants and suicide attempts: an analysis of the Veterans Health Administration data sets. Am J Psychiatry 2007:164:1044-9.

3. Søndergård L, Kvist K, Andersen PK, et al. Do antidepressants precipitate youth suicide? A nationwide pharmacoepidemiological study. Eur Child Adolesc Psychiatry 2006;15:232-40.

4. Gibbons RD, Hur K, Bhaumik DK, et al. The relationship between antidepressant prescription rates and rate of early adolescent suicide. Am J Psychiatry 2006; 163:1898-904.

5. Olfson M, Shaffer D, Marcus SC, et al. Relationship between antidepressant medication treatment and suicide in adolescents. Arch Gen Psychiatry 2003:60:978-82.

6. Gibbons RD, Brown CH, Hur K, et al. Early evidence on the effects of regulators suicidality warnings on SSRI prescriptions and suicide in children and adolescents. Am J Psychiatry 2007;164:1356-63.

7. Bridge JA, Greenhouse JB, Weldon AH, et al. Suicide trends among youths aged 10 to 19 years in the United States, 1996-2005. JAMA 2008;300:1025-6.

8. Libby AM, Brent DA, Morrato EH, et al. Decline in treatment of pediatric depression after FDA advisory on risk of suicidality with SSRIs. Am J Psychiatry 2007; 164:884-91.

Correspondence to: Dr. J. John Mann, Molecular Imaging and Neuropathology, New York State Psychiatric Institute, Box 42, 1051 Riverside Dr., New York NY 10032, USA;

fax212 543-5652; jjm@columbia.edu

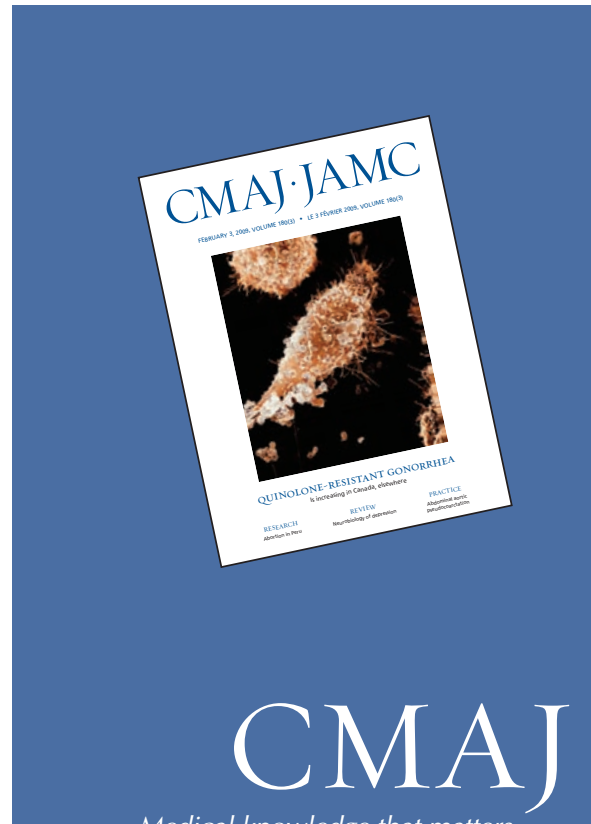

Medical knowledge that matters
3000 volunteer reviewers from around the world are the foundation for CMAJ's thorough, criteria-based review process. The editorial staff includes scientific consultants with expertise in statistics, experimental design and epidemiology.

To order your copy, call the CMA Member Service Centre tel $888855-2555$ or $613731-8610$ x2307 fax 613 236-8864 cmamsc@cma.ca

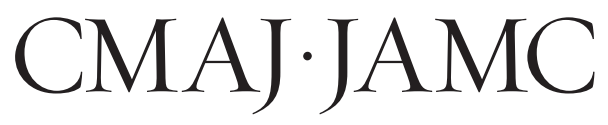

